\title{
A NEW MODEL FOR FUZZY PERSONALIZED ROUTE PLANNING USING FUZZY LINGUISTIC PREFERENCE RELATION
}

\author{
S. Nadi ${ }^{\text {a }}$ A. H. Houshyaripour ${ }^{b}$ \\ a Assistant Professor, Department of Geomatics Engineering, Faculty of Civil and Transportation Engineering, University of Isfahan, \\ Isfahan, Iran - snadi@eng.ui.ac.ir \\ ${ }^{\mathrm{b}}$ M.Sc. Remote Sensing, Department of Geomatics Engineering, Faculty of Civil and Transportation Engineering, University of \\ Isfahan, Iran - amirhossein7jm@gmail.com
}

KEY WORDS: Fuzzy Route Planning, Personalized, Uncertainty, Fuzzy AHP, Fuzzy Comparison

\begin{abstract}
:
This paper proposes a new model for personalized route planning under uncertain condition. Personalized routing, involves different sources of uncertainty. These uncertainties can be raised from user's ambiguity about their preferences, imprecise criteria values and modelling process. The proposed model uses Fuzzy Linguistic Preference Relation Analytical Hierarchical Process (FLPRAHP) to analyse user's preferences under uncertainty. Routing is a multi-criteria task especially in transportation networks, where the users wish to optimize their routes based on different criteria. However, due to the lake of knowledge about the preferences of different users and uncertainties available in the criteria values, we propose a new personalized fuzzy routing method based on the fuzzy ranking using center of gravity. The model employed FLPRAHP method to aggregate uncertain criteria values regarding uncertain user's preferences while improve consistency with least possible comparisons. An illustrative example presents the effectiveness and capability of the proposed model to calculate best personalize route under fuzziness and uncertainty.
\end{abstract}

\section{INTRODUCTION}

Personalized routing, involves different sources of uncertainty. These uncertainties can be raised from user's ambiguity about their preferences, imprecise criteria values and modeling process. To find the best personalized route the model should have the flexibility to consider all these sources of uncertainty. To cope with this problem, integration of routing methods with fuzzy decision theory is an idea that is followed in this article.

During the recent years, a number of researches have been done in personalized routing (Vahidinia et.al., 2008, Khan and Alnuweiri, 2004, Rodriquez and Lazo, 2013). Teodorovic and Kilkuchi (2007) presented a model follows the principles of the classical Clarke-Wright algorithm to develop a set of vehicle routes (Teodorovic and Kilkuchi, 2007). Khan and Alnuweiri (2004) proposed a low-complexity constraint-based routing algorithm for traffic engineering in computer networks that route end-to-end packet flows (Khan and Alnuweiri, 2004). The proposed fuzzy routing algorithm (FRA), modifies the wellknown Dijkstra's single-source shortest paths algorithm by including fuzzy membership functions in the path-cost update process (Khan and Alnuweiri, 2004). Zheng and Liu (2004) considered the vehicle routing problem with the travel times as the fuzzy variables. Then they designed a fuzzy optimization model for fuzzy vehicle routing based on the time window (Zheng and Liu, 2004). Boyan and Littman (1994) described the Q-routing algorithm for packet routing in computer networks. They embedded a reinforcement learning into each node of a switching network. In their model each node only used local communication to keep minimal delivery times (Boyan and Littman, 1994).

In this paper we propose a new model for finding the best personalized route based on the fuzzy decision theory, taking into account the uncertainties in either the measurements or preferences to provide users the ability to include their preferences in routing task easily, we propose a to use fuzzy linguistic preference relation AHP method (FLPRAHP). The most challenging issues in including user's preferences in any personalized systems using multi-criteria methods are the number of comparisons, consistency of comparisons and ambiguities in presenting the exact preferences. The FLPRAHP provide a mechanism for improving consistency using least number of comparisons and include user's linguistic phrases about their preferences (Wang and Chen, 2008). The FLPRAHP provides costs of links as fuzzy numbers. To find the best route regarding these fuzzy costs, we proposed a new fuzzy routing method based on incorporation of Center of Gravity Fuzzy Comparison (CGFC) method and dijkstra's algorithm. An illustrative example is then used to present the application of the proposed method.

The paper is organized as follows. In section 2 we present the proposed methodology describing FLPRAHP, center of gravity fuzzy comparison method and their incorporation in Dijkstra's algorithm. In section 3 we detail an illustrative example showing the step by step application of the proposed method. Some conclusions and future directions of the work are included in section 4 .

\section{METODOLOGY}

As illustrated in figure 1, the proposed model consists of 2 steps. In the first step, the model provides the basis for capturing users preferences which are always uncertain. The most challenging issues in this step are reducing the number of comparisons between criteria to determine user's preferences and maintaining the comparisons consistent. To cope with these problems, in this step the fuzzy linguistic preference relation method is adopted. This step results in a set of fuzzy numbers represents the cost of each link in the network. The second step uses these fuzzy costs for each link of the network as input values for fuzzy 
personalized routing engine to solve the best path problem. We design and develop fuzzy personalized routing engine by integrating fuzzy center of gravity ranking method with dijkstra's algorithm.

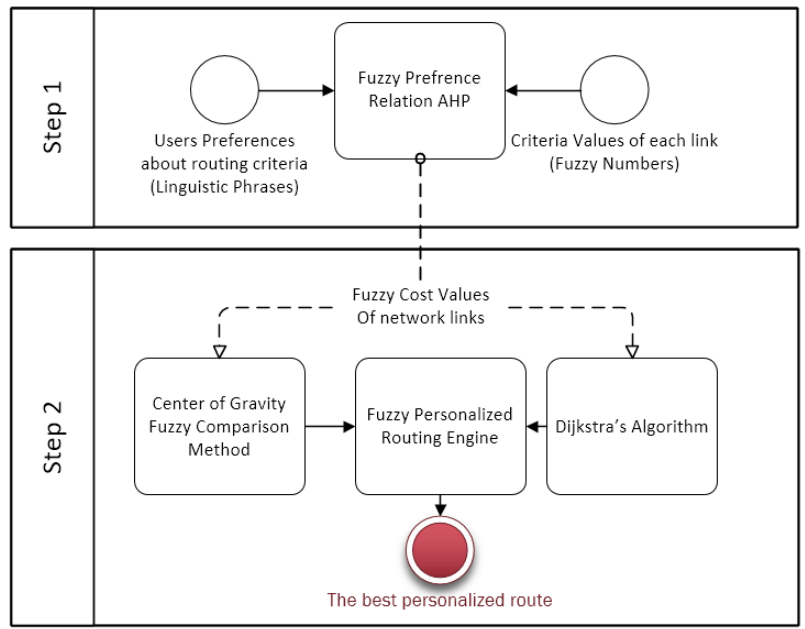

Figure 1. Fuzzy personalized routing model

\subsection{Conventional Fuzzy AHP}

The Analytical Hierarchical Process (AHP) is one of the extensively used multi-criteria decision making methods. Although this method is easy to understand and it can model expert opinions through pairwise comparison, however, the conventional AHP cannot process imprecise or vague information (Chen and Hwang, 1991, Chiclana and Herrera, 1998, Vahidnia et.al, 2008, Zhu, 2014) in conventional AHP decision makers compare criteria using crisp judgments. However, in the real situations most experts can just use their judgments regarding criteria relative meaning which are usually vague. It is the essence of the AHP that human judgments, and not just the underlying information, can be used in performing the evaluations. To model the ambiguity in judgments and also uncertainty in criteria values fuzzy extensions of AHP has been introduced. However, the most challenging issue of these methods are to maintain the comparisons consistence. The FLPRAHP provides a method to capture the experts' preferences about criteria using fuzzy linguistic phrases and calculates importance weight of each criterion using least possible number comparison while maintains consistency. The steps of the conventional Fuzzy AHP are as follows:

Step 1: Hierarchical structure construction by placing the goal of the desired problem on the top level of the hierarchical structure, the evaluation criteria on the middle levels and the alternatives on the bottom level.

Step 2: Constructing the fuzzy judgment matrix $\widetilde{A}$. The fuzzy judgment matrix $\widetilde{A}$ in equation 1 is a pairwise comparison of criteria that is constructed by assigning linguistic terms to the pairwise comparisons by asking which one of two criteria is more important.

$\tilde{A}=\left[\begin{array}{cccc}\tilde{1} & \tilde{a}_{12} & \ldots & \tilde{a}_{1 n} \\ \tilde{a}_{21} & \tilde{1} & \cdots & \tilde{a}_{2 n} \\ \vdots & \vdots & \ddots & \vdots \\ \tilde{a}_{n 1} & \tilde{a}_{n 2} & \cdots & \tilde{1}\end{array}\right]=\left[\begin{array}{cccc}\tilde{1} & \tilde{a}_{12} & & \tilde{a}_{1 n} \\ \tilde{a}_{12}{ }^{-1} & \tilde{1} & & \tilde{a}_{2 n} \\ \vdots & \vdots & \ddots & \vdots \\ \tilde{a}_{1 n}{ }^{-1} & \tilde{a}_{2 n}{ }^{-1} & \cdots & \tilde{1}\end{array}\right]$

where $\tilde{a}_{i j}$ is the fuzzy number from table 1 resulted by comparing $i$ th and $j$ th criteria.
Table 1. Membership function of linguistic scales

\begin{tabular}{l|l|l} 
Fuzzy numbers & Linguistic scales & Membership function \\
\hline$\widetilde{\mathbf{1}}$ & Equally important & $(1,1,3)$ \\
$\widetilde{\mathbf{3}}$ & Weakly important & $(1,3,5)$ \\
$\widetilde{\mathbf{5}}$ & Essentially important & $(3,5,7)$ \\
\hline$\widetilde{\mathbf{7}}$ & Very strong important & $(5,7,9)$ \\
\hline$\widetilde{\mathbf{9}}$ & Absolutely important & $(7,9,9)$ \\
\hline$\widetilde{\mathbf{1}}^{\mathbf{1}} \cdot \widetilde{\mathbf{3}}^{-\mathbf{1}} \cdot \widetilde{\mathbf{5}}^{\mathbf{- 1}} \cdot \widetilde{\mathbf{7}}^{-\mathbf{1}} \cdot \widetilde{\mathbf{9}}^{-\mathbf{1}}$ & Relative less important & $\ldots$ \\
\hline
\end{tabular}

Step 3: Calculating fuzzy weights of each criterion. The fuzzy weights of each criterion are calculated using equation 2 (Ekel et.al., 2006).

$\widetilde{w}_{i}=\frac{\tilde{r}_{i}}{\tilde{r}_{1} \oplus \ldots \oplus \tilde{r}_{n}}$

$\tilde{r}_{i}=\left[\tilde{a}_{i 1} \otimes \tilde{a}_{i 2} \otimes \ldots \otimes \tilde{a}_{i n}\right]^{\frac{1}{n}} \quad \forall i=1,2, \ldots, n$

Where $\widetilde{w}_{i}$ is the importance weights of $i$ th criterion.

Step 4: Hierarchical layer sequencing. The final fuzzy weight value of each alternative is calculated by hierarchical layer sequencing using equation 3 .

$\widetilde{U}_{i}=\sum_{j=1}^{n} \widetilde{w}_{j} \cdot \tilde{r}_{i j} \quad . \widetilde{U}_{i}=($ l.m.u $)$

Where $\tilde{r}_{i j}$ is the fuzzy value of the $j$ th criterion, $\widetilde{U}_{i}$ is a fuzzy number shows the final score of $i$ th criterion.

Step 5: Ranking alternatives.

To prepare alternative for ranking at the final step, one approach is defuzzification which transform fuzzy numbers to crisp ones. Equation 4 shows one of the simplest but least useful methods named weighted fuzzy mean.

$X\left(\widetilde{U}_{i}\right)=(l+m+u) / 3$

Where $1, \mathrm{~m}$ and $\mathrm{u}$ are lower, mid and upper band of fuzzy number $\widetilde{U}_{i}$ and $X\left(\widetilde{U}_{i}\right)$ is fuzzy mean of $\widetilde{U}_{i}$ which can be used to determine the optimum alternative.

In this paper we propose to use fuzzy ranking method of center of gravity that uses more knowledge from fuzzy numbers to compare them. Furthermore, when the number of criteria become large the number of comparisons as well as maintaining them consistent would be a challenging issue especially in personalized systems where public users are supposed to make comparisons. Here we propose to use FLPRAHP methods in personalized systems which solve these problems. Following we explain the FLPRAHP and Fuzzy Ranking methods in sections 2.2 and 2.3 respectively.

\subsection{Fuzzy Linguistic preference relation AHP}

In the second step of conventional fuzzy AHP described in section 2.1, the amount of comparison can be reduced using the relationship between elements of the matrix $\widetilde{C}$ (Berredo et.al., 2005, Ekel et.al., 2006, Wang and Chen, 2008). Given that the fuzzy positive matrix $\tilde{A}=\left(\tilde{a}_{i j}\right)$ is reciprocal which means that $\tilde{a}_{j i}=\tilde{a}_{i j}{ }^{-1}$ where $\tilde{a}_{i j} \in[1 / 9.9]$ the fuzzy preference relation matrix $\tilde{P}=\left(\tilde{p}_{i j}\right)$ where $\tilde{p}_{i j} \in[0.1]$ can be calculated using transformation in equation 5 (Ekel et.al., 2006).

$\tilde{p}_{i j}=\frac{1}{2}\left(1+\log _{9} a_{i j}\right) \quad \cdot \quad \tilde{p}_{i j}=\left(p_{i j}^{L} \cdot p_{i j}^{M} \cdot p_{i j}^{R}\right)$

Where $p_{i j}^{L}, p_{i j}^{M}$ and $p_{i j}^{R}$ are left, mid and right band of fuzzy number $\tilde{p}_{i j}$.

For $\tilde{A}=\left(\tilde{a}_{i j}\right)$ being consistent, $\tilde{a}_{i k}$ should be equal to $\tilde{a}_{i j} \otimes \tilde{a}_{j k}$. Taking logarithm on both sides equation 6 yields (Ekel et.al., 2006).

$\tilde{a}_{i j} \otimes \tilde{a}_{j k} \cong \tilde{a}_{i k}$ 
$\log _{9} a_{i j} \oplus \log _{9} a_{j k}=\log _{9} a_{i k}$

$\log _{9} a_{i j} \oplus \log _{9} a_{j k} \ominus \log _{9} a_{i k}=0$

$\log _{9} a_{i j} \oplus \log _{9} a_{j k} \oplus \log _{9} a_{k i}=0$

$\frac{1}{2}\left(1+\log _{9} a_{i j}\right) \oplus \frac{1}{2}\left(1+\log _{9} a_{j k}\right) \oplus \frac{1}{2}\left(1+\log _{9} a_{k i}\right)=\frac{3}{2}$

Substituting $\frac{1}{2}\left(1+\log _{9} a_{i j}\right)$ from equation 5 the relationship between elements of matrix $\tilde{A}$ can be determined as detailed in equation 7 (Ekel et.al., 2006).

$\tilde{p}_{i j} \oplus \tilde{p}_{j k} \oplus \tilde{p}_{k i}=\frac{3}{2}$
$p_{i j}^{L}+p_{j k}^{L}+p_{k i}^{R}=\frac{3}{2}$
$p_{i j}^{M}+p_{j k}^{M}+p_{k i}^{M}=\frac{3}{2}$
$p_{i j}^{R}+p_{j k}^{R}+p_{k i}^{L}=\frac{3}{2}$

These relationships for more than three criteria are as equation 8 (Ekel et.al., 2006).

$p_{i(i+1)}^{L}+p_{(i+1)(i+2)}^{L}+\cdots+p_{(j-1) j}^{L}+p_{j i}^{R}=\frac{(j-i+1)}{2}$

$p_{i(i+1)}^{M}+p_{(i+1)(i+2)}^{M}+\cdots+p_{(j-1) j}^{M}+p_{j i}^{M}=\frac{(j-i+1)}{2}$

$p_{i(i+1)}^{R}+p_{(i+1)(i+2)}^{R}+\cdots+p_{(j-1) j}^{R}+p_{j i}^{L}=\frac{(j-i+1)}{2}$

By using these relationships, the required comparisons for $\mathrm{n}$ criteria will be reduced from $n(n-1) / 2$ to just $n-1$ comparisons while the consistency is maintained.

\subsection{Centre of Gravity Fuzzy Ranking Method}

There are many ways to compare two fuzzy numbers. Center of gravity is one of the most common and useful techniques (Chen and Chen, 2009, Chen and Chen, 2007, Chan and Qi, 2002, Phani and Shankar, 2011).

Step 1: Considering $\tilde{A}_{i}=\left(a^{L} \cdot a^{M 1} \cdot a^{M 2} \cdot a^{R} \cdot w_{\tilde{A}_{i}}\right)$ as a generalized trapezoidal fuzzy number where $a^{L}, a^{M 1}, a^{M 2}$ and $a^{R}$ are real numbers and $0<w_{\tilde{A}_{i}} \leq 1$ is its maximum membership value. In this step the standardized trapezoidal fuzzy number must be calculated using 9 .

$$
\begin{aligned}
\tilde{A}_{i}^{*}=\left(\frac{a^{L}}{k}, \frac{a^{M 1}}{k}, \frac{a^{M 2}}{k}\right. & \left., \frac{a^{R}}{k} ; w_{\tilde{A}_{i}}\right) \\
& =\left(a^{L^{*}}, a^{M 1^{*}}, a^{M 2^{*}}, a^{R^{*}} ; w_{\tilde{A}_{i}}\right)
\end{aligned}
$$

Where $k=\max \left(a^{L} \cdot a^{M 1} \cdot a^{M 2} \cdot a^{R}, 1\right)$ and $0 \leq a^{L^{*}} \leq a^{M 1^{*}} \leq$ $a^{M 2^{*}} \leq a^{R^{*}} \leq 1$.

Step 2: Computing the Centre of gravity $\left(x_{\tilde{A}_{i}}^{*} \cdot y_{\tilde{A}_{i}}^{*}\right)$ using equation 10 (Chen and Chen, 2009).

$$
\begin{aligned}
& y_{\tilde{A}_{i}}^{*}= \begin{cases}\frac{w_{\tilde{A}_{i}}\left(\frac{a^{M 2^{*}}-a^{M 1^{*}}}{a^{R^{*}}-a^{L^{*}}}+2\right)}{6} \cdot & \text { if } a^{L^{*}} \neq a^{R^{*}} \\
\frac{w_{\tilde{A}_{i}}}{2} & \text { if } a^{L^{*}}=a^{R^{*}}\end{cases} \\
& x_{\tilde{A}_{i}}^{*}=\frac{y_{\tilde{A}_{i}}^{*}\left(a^{M 1^{*}}+a^{M 2^{*}}\right)+\left(a^{M 1^{*}}+a^{R^{*}}\right)\left(w_{\tilde{A}_{i}}-y_{\tilde{A}_{i}}^{*}\right)}{2 w_{\tilde{A}_{i}}}
\end{aligned}
$$

Step 3: Computing the mean and standard deviation (Chen and Chen, 2009)

$$
\bar{x}_{\tilde{A}_{i}}=\frac{a^{L^{*}}+a^{M 1^{*}}+a^{M 2^{*}}+a^{R^{*}}}{4}
$$

$S_{\tilde{A}_{i}}=\sqrt{\frac{\sum\left(x_{j}-\bar{x}_{\tilde{A}_{i}}\right)^{2}}{4-1}} \quad \cdot x_{j}=\left\{a^{L^{*}}, a^{M 1^{*}}, a^{M 2^{*}}, a^{R^{*}}\right\}$

Step 4: Computing rank of the standard fuzzy number using equation 12 (Chen and Chen, 2009).

$\operatorname{Rank}\left(\tilde{A}_{i}^{*}\right)=x_{\tilde{A}_{i}}^{*}+\left(w_{\tilde{A}_{i}}-y_{\tilde{A}_{i}}^{*}\right)^{S_{\widetilde{A}_{i}}} \times\left(y_{\tilde{A}_{i}}^{*}+0.5\right)^{1-w_{\tilde{A}_{i}}}$

This rank then could be used to rank fuzzy numbers. This approach can also be used for triangular fuzzy numbers by considering $a^{M 1}=a^{M 2}$ in the equations.

\subsection{Fuzzy Personalized Routing Engine}

The route planning algorithms determine a path through a network from an origin to a destination. For determination of this path, the corresponding minimization problem, over an impedance function, has to be solved. This paper proposes that the impedance of each link should be calculated according to the users' preferences using fuzzy linguistic preference relation AHP method as described in section 2.2. The route planning in a network with non-negative crisp costs of links can be solved easily using well-known existing approaches such as Dijkstra's labeling algorithm. However, when the costs of links are in the form of fuzzy numbers, as in the case in this paper, a fuzzy routing approach is required. We propose to adopt Dijkstra's algorithm for fuzzy numbers based on the fuzzy center of gravity ranking method which is described in section 2.3. The advantage of this approach over other existing approaches, which work only for acyclic, layered graphs, is that it is usable for any general directed graphs, including transportation networks.

Figure 2 illustrates the proposed algorithm for calculating the fuzzy personalized least cost route. In this approach, a directed graph $\mathrm{G}=(\mathrm{V}, \mathrm{A})$ is defined with node set $\mathrm{V}$, arc set $\mathrm{A}$, start node (origin) $\mathrm{o} \in \mathrm{V}$, target node (destination) $\mathrm{d} \in \mathrm{V}$ and cost_of_travelling $[u . v]=\left(L_{u v} \cdot M_{u v} \cdot R_{u v}\right)$ is fuzzy cost of travelling from node $\mathrm{u} \in \mathrm{V}$ to node $\mathrm{v} \in \mathrm{V}$ which share a link and $0 \leq \mathrm{L}_{\mathrm{uv}} \leq \mathrm{M}_{\mathrm{uv}} \leq \mathrm{U}_{\mathrm{uv}} \leq 1$.

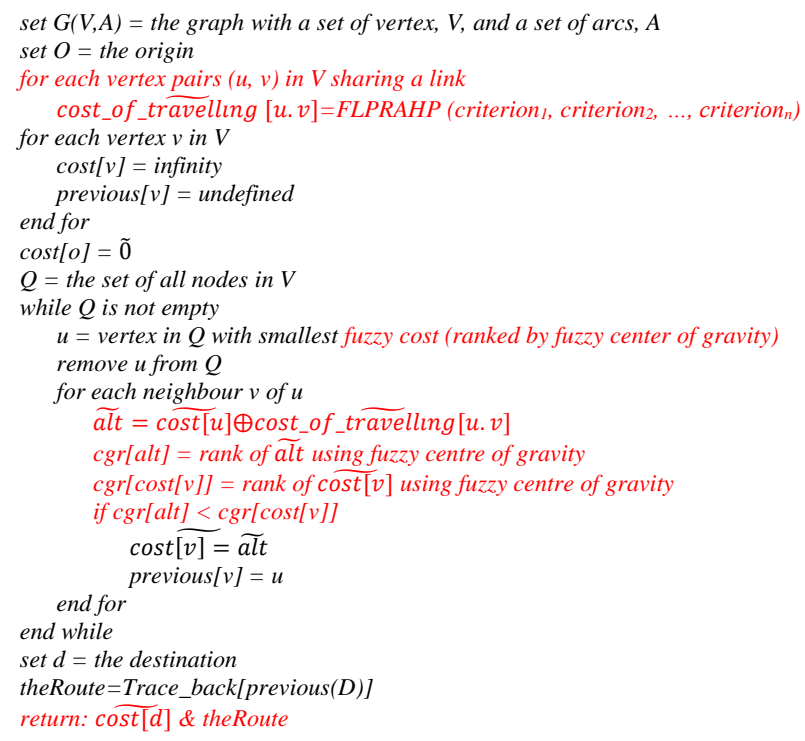

Figure 2. The proposed algorithm for determining personalized routing algorithm 


\section{NUMERICAL EXAMPLE}

Figure 3 shows the network to be evaluated and Table 2 illustrates the attributes of each link. The personalized fuzzy route between the origin, $\mathrm{A}$, and the destination, $\mathrm{F}$, are required for different decision strategies. In this example we supposed that the user is interested to find the best route regarding the "distance", "traffic volume" and "quality of road".

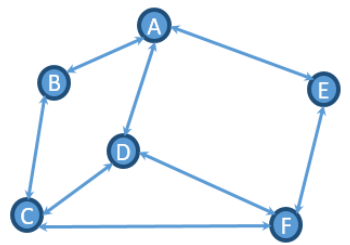

Figure 3. Sample network to be evaluated

Table 2. Fuzzy values of different criteria each link

\begin{tabular}{l|c|c|c} 
link & Road Distance & Road Quality & Traffic Volume \\
\hline $\mathrm{AB}$ & $\tilde{3}=(1.3 .5)$ & $\tilde{1}=(-1.1 .3)$ & $\tilde{5}=(3.5 .7)$ \\
\hline $\mathrm{AD}$ & $\tilde{4}=(2.4 .6)$ & $\tilde{2}=(0.2 .4)$ & $\tilde{4}=(2.4 .6)$ \\
\hline $\mathrm{AE}$ & $\tilde{5}=(3.5 .7)$ & $\tilde{2}=(0.2 .4)$ & $\tilde{6}=(4.6 .8)$ \\
\hline $\mathrm{BC}$ & $\tilde{3}=(1.3 .5)$ & $\tilde{1}=(-1.1 .3)$ & $\tilde{4}=(2.4 .6)$ \\
\hline $\mathrm{CD}$ & $\tilde{1}=(-1.1 .3)$ & $\tilde{0}=(-2.0 .2)$ & $\tilde{3}=(1.3 .5)$ \\
\hline $\mathrm{CF}$ & $\tilde{6}=(4.6 .8)$ & $\tilde{3}=(1.3 .5)$ & $\tilde{1}=(-1.1 .3)$ \\
\hline $\mathrm{DF}$ & $\tilde{2}=(0.2 .4)$ & $\tilde{1}=(-1.1 .3)$ & $\tilde{2}=(0.2 .4)$ \\
\hline $\mathrm{EF}$ & $\tilde{3}=(1.3 .5)$ & $\tilde{2}=(0.2 .4)$ & $\tilde{2}=(0.2 .4)$ \\
\hline
\end{tabular}

Regarding the method described in section 2, the following steps have been followed.

Step 1. Determining users' preferences:

Regarding the hierarchical tree illustrated in figure 4 there are 3 criteria namely "traffic volume", "road distance" and "road quality" in which we first should determine user preferences regarding them.

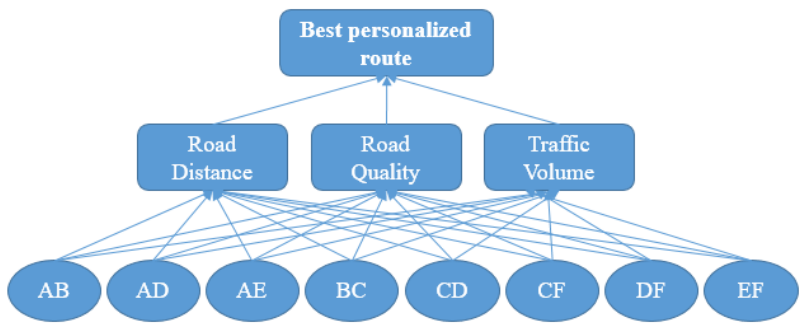

Figure 4. AHP hierarchical structure

Therefore, the first step goes through the process of completing the pair-wise comparison matrix and determining the user preferences using fuzzy linguistic preference relation AHP explained in section 2.2. To do this, the user just completed the first row of pair-wise comparison matrix based on linguistic preference relation in table 1 . Other comparisons can be obtained using equation 8 . Table 3 shows the comparisons by user.

Table 3. Fuzzy pair-wised comparison

\begin{tabular}{c|c|c|c} 
A & Distance & HVT & Traffic \\
\hline Distance & $\tilde{1}$ & $\tilde{3}$ & $\tilde{5}$ \\
\hline HVT & $?$ & $\tilde{1}$ & $?$
\end{tabular}

\begin{tabular}{|l|l|l|l|}
\hline Traffic & $?$ & $?$ & $\tilde{1}$ \\
\hline
\end{tabular}

Fuzzy preference relation value $P$ is calculated based on the equation 5 as showed in table 4 .

Table 4. Fuzzy preference relation of comparisons

\begin{tabular}{c|c|c|c}
$\mathrm{P}$ & Distance & HVT & Traffic \\
\hline Distance & $\tilde{1}$ & $(0.50 .750 .87)$ & $(0.750 .870 .94)$ \\
\hline HVT & $(0.130 .250 .50)$ & $\tilde{1}$ & $(0.570 .620 .75)$ \\
\hline Traffic & $(0.060 .130 .25)$ & $(0.250 .380 .43)$ & $\tilde{1}$
\end{tabular}

Having this matrix in hand, the weights of each criterion is calculated using equation 2 as follow.

$r($ road distance $)=\left[\begin{array}{lll}0.72 & 0.87 & 1.35\end{array}\right]$

$r($ road quality $)=\left[\begin{array}{lll}0.42 & 0.54 & 1.04\end{array}\right]$

$r($ traffic volume $)=\left[\begin{array}{lll}0.25 & 0.37 & 0.69\end{array}\right]$

$w_{1}=w($ road distance $)=\left[\begin{array}{lll}0.23 & 0.49 & 0.97\end{array}\right]$

$w_{2}=w($ road quality $)=\left[\begin{array}{lll}0.14 & 0.30 & 0.75\end{array}\right]$

$w_{3}=w($ traffic volume $)=\left[\begin{array}{lll}0.08 & 0.21 & 0.50\end{array}\right]$

Step 2. Calculating overall cost of each link:

By aggregating users' preferences regarding each criteria and their values for each link the overall cost of each link is calculated as follow.

cost_of_travellıng $[A . B]=\tilde{3} \otimes w_{1} \oplus \tilde{1} \otimes w_{2} \oplus \tilde{5} \otimes w_{3}$

$$
=\left[\begin{array}{lll}
0.78 & 2.82 & 8.38
\end{array}\right]=\overline{2.82}
$$

cost_of_travellıng $[$ A.E $]=\tilde{5} \otimes w_{1} \oplus \tilde{2} \otimes w_{2} \oplus \tilde{6} \otimes w_{3}$

$$
=\left[\begin{array}{lll}
1.46 & 4.31 & 11.57
\end{array}\right]=\overline{4.31}
$$

cost_of_travelling $[A . D]=\tilde{4} \otimes w_{1} \oplus \tilde{2} \otimes w_{2} \oplus \tilde{4} \otimes w_{3}$

$$
=\left[\begin{array}{lll}
1.07 & 3.40 & 9.60
\end{array}\right]=\overline{3.40}
$$

cost_of_travelling $[$ B.C $]=\tilde{3} \otimes w_{1} \oplus \tilde{1} \otimes w_{2} \oplus \tilde{4} \otimes w_{3}$

$$
=\left[\begin{array}{lll}
0.70 & 2.61 & 7.88
\end{array}\right]=\overline{2.61}
$$

cost_of_travelling $[D . C]=\tilde{1} \otimes w_{1} \oplus \tilde{0} \otimes w_{2} \oplus \tilde{3} \otimes w_{3}$

$$
=\left[\begin{array}{lll}
0.16 & 1.12 & 3.94
\end{array}\right]=\overline{1.12}
$$

cost_of_travelling $[E . F]=\tilde{3} \otimes w_{1} \oplus \tilde{2} \otimes w_{2} \oplus \tilde{2} \otimes w_{3}$

$$
=\left[\begin{array}{lll}
0.68 & 2.49 & 7.63
\end{array}\right]=\overline{2.49}
$$

cost_of_travellıng $[D . F]=\tilde{2} \otimes w_{1} \oplus \tilde{1} \otimes w_{2} \oplus \tilde{2} \otimes w_{3}$

$$
=\left[\begin{array}{lll}
0.31 & 1.70 & 5.91
\end{array}\right]=\overline{1.70}
$$

cost_of_travellıng $[$ C.F $]=\tilde{6} \otimes w_{1} \oplus \tilde{3} \otimes w_{2} \oplus \tilde{1} \otimes w_{3}$

$$
=\left[\begin{array}{lll}
1.43 & 4.05 & 10.79
\end{array}\right]=\overline{4.04}
$$

Figure 5 illustrates the resulted network with overall cost of each link.

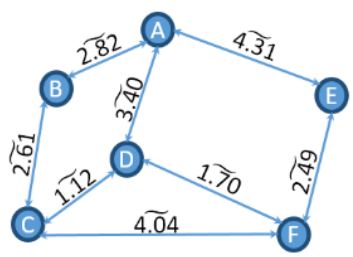

Figure 5. overall fuzzy cost of each link

Step 3. Finding the best path using fuzzy personalized routing method:

In the final step, fuzzy personalized routing engine, which has explained in section 2.4, uses fuzzy cost of travelling on each link to find the best route. The data structure in Table 5 shows the steps for determining best route.

Table 5. the proposed data structure to determine optimum personalized route
\begin{tabular}{|c|c|c|c|c|c|c|c|c|c|c|c|c|c|c|c|c|c|c|c|}
\hline \\
\cline { 2 - 20 } \\
Vertex loop & 11 & 12 & 13 & 14 & 15 & L6 & 11 & 12 & 13 & 14 & 15 & L6 & 11 & 12 & 13 & 14 & 15 & L6 \\
\hline A & 0 & - & - & - & - & - & - & - & - & - & - & - & $\checkmark$ & - & - & - & - & - \\
\hline B & $\infty$ & 2.82 & - & - & - & - & Null & A & - & - & - & - & & $\checkmark$ & - & - & - & - \\
\hline C & $\infty$ & $\infty$ & 5.43 & 4.52 & 4.52 & - & Null & Null & B & D & D & - & & & & & $\checkmark$ & - \\
\hline
\end{tabular}




\begin{tabular}{|c|c|c|c|c|c|c|c|c|c|c|c|c|c|c|c|c|c|c|}
\hline D & $\infty$ & $\overline{3.40}$ & $\overline{3.40}$ & - & - & - & Null & A & A & - & - & - & & & $\checkmark$ & - & - & - \\
\hline E & $\infty$ & $\overline{4.31}$ & $\overline{4.31}$ & $\overline{4.31}$ & - & - & Null & A & A & A & - & - & & & & $\checkmark$ & - & - \\
\hline F & $\infty$ & $\infty$ & $\infty$ & $\overline{5.10}$ & $\widetilde{6.8}$ & $\overline{8.56}$ & Null & Null & Null & D & E & C & & & & & & $\checkmark$ \\
\hline
\end{tabular}

In this table, $11,12, \ldots, 16$ columns preserve the results of the steps in routing algorithm which are illustrated in figure 2 . For the sample network illustrated in figure 3 the algorithm determined the best path in 6 loops in this table. Table 6 shows the required comparisons for each loop using fuzzy center of gravity.

Table 6. required comparisons in each loop

\begin{tabular}{|c|c|c|c|c|c|c|c|}
\hline oop number & Izzy numbers & & $x^{*}$ & $y^{*}$ & $\bar{x}$ & $s$ & Rank \\
\hline \multirow{3}{*}{17} & {$\left[\begin{array}{ll}0.78 & 2.82\end{array}\right.$} & $8.38]=2 . \widetilde{8} 2$ & 3.93 & 0.37 & 3.99 & 3.28 & 4.157 \\
\hline & {$[1.07$} & $9.60]=3 . \widetilde{4} 0$ & 4.61 & 0.38 & 4.69 & 3.68 & 4.785 \\
\hline & {$\left[\begin{array}{ll}1.46 & 4.31\end{array}\right.$} & $11.57]=4 . \widetilde{3} 1$ & 5.65 & 0.39 & 5.78 & 4.34 & 5.764 \\
\hline & [1.48 & $16.26]=\overline{5.43}$ & 7.61 & 0.36 & 7.72 & 6.39 & 7.671 \\
\hline & {$\left[\begin{array}{ll}1.07 & 3.40\end{array}\right.$} & $9.60]=3 . \widetilde{4} 0$ & 4.61 & 0.38 & 4.69 & 3.68 & 4.784 \\
\hline & {$[1.46$} & $11.57]=4.31$ & 5.65 & 0.39 & 5.78 & 4.34 & 5.764 \\
\hline & {$[1.23$} & & 6.34 & 0.36 & 6.43 & 5.32 & 6.430 \\
\hline & {$\left[\begin{array}{ll}1.46 & 4.31\end{array}\right.$} & $=4.31$ & 5.65 & 0.39 & 5.78 & 4.34 & 5.764 \\
\hline & {$\left[\begin{array}{ll}1.38 & 5.1\end{array}\right.$} & $15.51]=\widetilde{5.1}$ & 7.25 & 0.36 & 7.33 & 6.12 & 7.317 \\
\hline & {$[1.23$} & $13.54]=\widetilde{4.52}$ & 6.34 & 0.36 & 6.43 & 5.32 & 6.430 \\
\hline & {$[2.14$} & $19.20]=\overline{6.80}$ & 9.22 & 0.38 & 9.38 & 7.35 & 9.247 \\
\hline
\end{tabular}

To find the path to any vertex in the network, one can find the minimum cost value in its row and trace back using the previous vertex named in the same loop in "Previous vertex" columns. For example for $\mathrm{f}$ as the destination minimum cost belongs to loop 4 with $\widetilde{5.10}$ which is related to its previous vertex $\mathrm{D}$ in loop 4 . Then, for vertex $\mathrm{D}$ the minimum cost belongs to loop 3 with $\overline{3.40}$ which is related to its previous vertex A in this loop. Therefore, the best path to $\mathrm{F}$ would be $\mathrm{A} \rightarrow \mathrm{D} \rightarrow \mathrm{F}$. it should be explained that the next best paths to $F$ with $\widetilde{6.8}$ and $\widetilde{8.56}$ cost values can be determined by tracing back from loops 5 and 6 respectively.

\section{CONCLUSION AND FUTURE DIRECTIONS}

Personalized route planning algorithms use different criteria of the network and aggregates them regarding users' preferences to determine overall cost for each link. These cost then are used to determine the best route. In this paper we emphasize that in this process there are two kinds of uncertainties. The first one is in the criteria values e.g. traffic, quality of the road and so on. Another one is from the ambiguity in determining users' preferences. Furthermore, the number of comparison between criteria to determine users' preferences as well as maintaining the consistency of comparisons are other important challenging issues. In this paper we propose a multi-criteria personalized routing model based on the fuzzy linguistic preference relation AHP model and modify Dijkstra's routing algorithm using fuzzy center of gravity ranking method to cope with this issues. Finally, we provide an illustrative example which shows the capability of the proposed model to capture the mentioned uncertainties.

\section{REFERENCES}

Berredo R. C., Ekel P. Y. and R. M. Palhares (2005). "Fuzzy preference relations in models of decision making", Nonlinear Analysis Theory, Methods \& Applications 63(5-7): 735-741

Boyan J. A. and M. L. Littman (1994) "Packet routing in dynamically changing networks: A reinforcement learning approach", In Cowan, J. D., Tesauro, G., and Alspector, J. (eds.), Advances in Neural Information Processing Systems 6(NIPS): 8 pages, Morgan Kaufmann.

Chan F. T. S. and H. J. Qi (2002). "A fuzzy basis channelspanning performance measurement method for supply chain management", Journal of Engineering Manufacture 216(8): $1155-1167$.

Chen S. J. and S. M. Chen (2009). "Fuzzy Risk Analysis Based on the Ranking of Generalized Trapezoidal Fuzzy Numbers", Applied Intelligence 26(1): 1-11.

Chen, S. M. and J. H. Chen (2007). "Fuzzy Risk Analysis Based on Ranking Generalized Fuzzy Numbers with Different Heights and Different Spreads", Expert Systems with Applications 36(3): 6833-6842.

Chen, S. J. and C. L. Hwang (1991). "Fuzzy Multiple Attribute Decision making", $\underline{\text { Springer }}$

Chiclana F. and E. Herrera-Viedma (1998). "Integrating three representation models in fuzzy multipurpose decision making based on fuzzy preference relations", Fuzzy Sets and Systems 97(1): 33-48.

Ekel P.Y., Silva M.R., Schuffner Neto F. and R.M. Palhares (2006). "Fuzzy preference modeling and its application to multiobjective decision making", Computers and Mathematics with Applications 52(1-2): 179-196.

Khan J. A. and H. M. Alnuweiri (2004). "A fuzzy constraintbased routing algorithm for traffic engineering", In Proc. of the Global Telecommunications Conference, GLOBECOM '04 3:1366- 1372, IEEE.

Phani Bushan Rao P. and N. Ravi Shankar (2011). "Ranking Fuzzy Numbers with a Distance Method using Circumcenter of Centroids and an Index of Modality", Advances in Fuzzy Systems Vol. 2011, 7pages.

Rodriguez-Puente R. and M. S. Lazo-Cortes (2013). "Algorithm for shortest path search in Geographic Information Systems by using reduced graphs", SpringerPlus 2(291): 13pages.

Teodorovic D. and S. Kikuchi (2007). "Application of fuzzy sets theory to the saving based vehicle routing algorithm", $\underline{\text { Civil }}$ Engneering Systems 8, 1991(2): 87-93.

Vahidniaa M.H., Alesheikh A., Alimohamadi A. and A. Basiri (2008). "Fuzzy analytical hierarchy process in GIS application", The International Archives of the Photogrammetry Remote Sensing and Spatial Information Sciences Vol. XXXVII part B2.

Wang T. C. and Y.H. Chen (2008). "Applying fuzzy linguistic preference relations to the improvement of consistency of fuzzy AHP", Information Sciences 178(19): 3755-3765.

Zheng Y. and B. Liu (2006). "Fuzzy vehicle routing model with credibility measure and its hybrid intelligent algorithm" Applied Mathematics and Computation 176(2): 673-683.

Zhu K. (2014). "Fuzzy analytic hierarchy process: Fallacy of the popular methods" European Journal of Operational Research 236(1): 209-217. 